Achtung

Bitte informieren Sie sich frühzeitig, ob der für Sie interessante Termin stattfindet und kontaktieren Sie bei Fragen direkt den Veranstalter.

\section{AUGUST 2020}

Amsterdam, Niederlande,

04.-08. August 2020

IASP 2020 World Congress on Pain

International Association for the Study of Pain (IASP)

wWw.iaspworldcongress.org

\section{SEPTEMBER 2020}

London, Großbritannien

11.-13. September 2020

13th European Congress for

Integrative Medicine

European Society of Integrative

Medicine (ECIM)

www.ecimcongress.com

Freudenstadt, 16.-20. September 2020 139. ZAEN-Kongress

Zentralverband der Ärzte für Naturheilverfahren und Regulationsmedizin e.V. www.zaen.org

Wiesbaden, 24.-26. September 2020 15. Deutscher Allergiekongress Ärzteverband Deutscher Allergologen e.V. (AeDA), Deutsche Gesellschaft für Allergologie und klinische Immunologie e.V. (DGAKI), Gesellschaft für Pädiatrische Allergologie und Umweltmedizin e.V. (GPA)

www.allergiekongress.de

Graz, Österreich,

24.-26. September 2020

17. Internationaler TCM Kongress

Stress- und Lifestyle-Erkrankungen in der TCM

Österreichische Gesellschaft für Kontrol-

lierte Akupunktur und TCM (OGKA)

www.tcmkongress.at

\section{OKTOBER 2020}

Mannheim, 21.-24. Oktober 2020 Deutscher Schmerzkongress

Deutsche Schmerzgesellschaft e.V.

Deutsche Migräne- und Kopfschmerzgesellschaft e.V.

Klinik für Neurologie, Universitätsklini-

kum Essen

www.schmerzkongress2020.de
Berlin, 23.-25. Oktober 2020

Training Acupuncturists without

\section{Borders (AWB)}

Kontakt über Dr. med. Elisabeth

Friedrichs (friedrichs@qigong-

yangsheng.de)

www.acuwithoutborders.org

München, 24. Oktober 2020

SYMPOSIUM Psychiatrie

Deutsche Ärztegesellschaft für

Akupunktur e.V. - DÄGfA in Kooperation

mit der LMU München

www.daegfa.de

\section{NOVEMBER 2020}

Berlin, 14. November 2020

SYMPOSIUM ,Wenn das Herz eng

wird"

Deutsche Ärztegesellschaft für

Akupunktur e.V. - DÄGfA

www.daegfa.de

Wien, Österreich, 20.-22. November 2020

Akupunkturkongress 2020

Gesunde Mitte - Akupunktur und TCM

für Darm und Mikrobiom

Ärzte für Akupunktur (ÄfA)

www.akupunktur.org

\section{FEBRUAR 2021}

Mannheim, 26.-27. Februar 2021

Geburtshilfe im Dialog \& TCM im

Dialog

Internationaler Kongress, Forum für den

Dialog in der Chinesischen Medizin

www.tcm-im-dialog.de

Berlin, 28. Februar - 02. März 2021 VegMed - Medizinischer Fachkongress zu pflanzenbasierter Ernährung ProVeg e.V., Physicians Association for Nutrition e.V., Immanuel Krankenhaus Berlin

www.vegmed.org

\section{MÄRZ 2021}

Freudenstadt, 17.-21. März 2021 140. ZAEN-Kongress Zentralverband der Ärzte für Naturheilverfahren und Regulationsmedizin e.V. www.zaen.org

\section{APRIL 2021}

Wien, Österreich, 10.-11. April 2021

Jahreskongress der ÖGA

8. Johannes Bischko Symposium Hormone - STATE OF THE ART meets

TCM

Österreichische Gesellschaft für

Akupunktur (ÖGA)

www.tcmsymposium.at
Erfurt, 15.-18. April 2021

39. DGfAN-Kongress

Alt werden - gesund bleiben

Deutsche Gesellschaft für Akupunktur

und Neuraltherapie e. V. (DGfAN)

www.dgfan.de

Hamburg, 17.-18. April 2021

4. Qigong-Kongress

Philosophie- und Praxistage

Verband für Turnen und Freizeit (VTF)

www.qigong-kongress.de

Chicago, Illinois, USA, 18.-20. April 2021

International Congress on Integrative

Medicine and Health

Academic Consortium for Integrative

Medicine and Health

www.imconsortium.org

Dortmund, 22.-24. April 2021

11. Dattelner Kinderschmerztag

Multiprofessioneller Kongress für

Kinderschmerztherapie und Pädiatrische

Palliativversorgung

www.kinderpalliativzentrum.de/

dattelner-kinderschmerztage

Hamburg, 22.-24. April 2021

Sports, Medicine and Health Summit

49. Deutscher Sportärztekongress

9th Congress of Exercise in Medicine

Europe

10. Internationaler Hamburger Sport-

Kongress

8. Symposium AG Diabetes Sport und

Bewegung

www.sports-medicine-health-summit.de

Hamburg, 24. April 2021

SYMPOSIUM Sport

Deutsche Ärztegesellschaft für

Akupunktur

e. V. - DÄGfA in Kooperation mit dem

Deutschen Sportärztekongress

www.daegfa.de

\section{MAI 2021}

Bad Nauheim, 13. Mai 2021 Jahrestagung der DÄGfA eingebettet in die 35. Internationale Akupunktur-Woche in Bad Nauheim Deutsche Ärztegesellschaft für Akupunktur e.V. (DÄGfA)

www.daegfa.de

Florence, Italien, 25.-28. Mai 2021

14th European Congress for

Integrative Medicine

2nd World Congress together with SCMR

www.ecim-congress.org

\section{JUNI 2021}

Pöllauberg, Österreich, 03--05. Juni 2021

25. Jahressymposium der OGKH

Österreichische Gesellschaft für

Kontrollierte Akupunktur (OGKA)

www.ogka.at/jahressymposium

\section{JULI 2021}

Heidelberg, 01.-03. Juli 2021

6. Heidelberger Tagung Neuralthe-

rapie

www.neuraltherapie-heidelberg.de

Athen, Griechenland, 02.-04. Juli 2021

34th ICMART World Congress on

Medical Acupuncture

International Council of Acupuncture

and Related Techniques

www.www.icmart2020.org

\section{SEPTEMBER 2021}

Heidelberg, 09.-12. September 2021

7th World Congress on Neural

Therapy

www.neuraltherapie-heidelberg.de

Montreal, Quebec, Kanada,

13.-15. September 2021

6th International Fascia Research

Congress

www.fasciacongress.org/2021-congress/

Freudenstadt, 22.-26. September 2021

141. ZAEN-Kongress

Zentralverband der Ärzte für Naturheil-

verfahren

und Regulationsmedizin e.V.

www.zaen.org

\section{OKTOBER 2021}

Tutzing, 08.-10. Oktober 2021

6. Kongress der SMS

Chinesische Medizin im klinischen

Alltag - Grundlagen, Anwendung \&

Wissenschaft

Internationale Gesellschaft für

chinesische Medizin (SMS)

www.tcm.edu/kongress/der-kongress

\section{NOVEMBER 2021}

Solothurn, Schweiz, 18.-20. November 2021

14. ASA-Kongress

Association Schweizer Ärztegesellschaft für Akupunktur und Chinesische Medizin (ASA)

www.asa-tcmkongress.ch 